\title{
Pleural Effusion in an Obese Lady with CoVid 19: A Challenging Case
}

\author{
Colaut $\mathrm{F}^{1 *}$, Piazza $\mathrm{A}^{1}$, Stecca $\mathrm{T}^{1}$, Pauletti $\mathrm{B}^{1}$, Greco $\mathrm{A}^{1}$, Romagnoli $\mathbf{M}^{2}$, \\ Lucernoni $\mathbf{P}^{2}$ and Massani $\mathbf{M}^{1}$ \\ ${ }^{1}$ 3rd General Surgery and Thoracic, Main Hospital Cà Foncello, Treviso, Italy \\ ${ }^{2}$ Pulmonology Department, Treviso, Italy
}

Case Report

Volume 5 Special Issue 1

Received Date: June 03, 2020

Published Date: July 01, 2020

*Corresponding author: Flavio Colaut, 3rd General Surgery and Thoracic, Main Hospital Cà Foncello, piazza ospedale 1, 31100, Treviso, Italy, Tel: +39 0422 3118844; Email: flavio.colaut@outlook.it

\section{Abstract}

CoVid-19 disease is a challenging health issue worldwide. This report describes the treatment of a huge pleural effusion on the right side in a lady positive to CoVid-19. Video Assisted Thoracic Surgery (VATS) was performed and she has come to an excellent clearance of the pleural space. Post-operative period was uneventful and now this lady has been discharged. Moreover, after intervention she is no longer positive to CoVid-19. Probably an early chest tube would have had avoided a surgical intervention in this case. Eventually it is reasonable to consider CoVid-19 pneumonia as a brand-new field of investigation about inflammatory pathways.

Keywords: CoVid 19; Pleural Effusion; VATS

Abbreviations: CoVid-19: Corona Virus disease 2019; VATS: Video Assisted Thoracic Surgery; C-XR: Chest X Ray; CT: Computed Tomography; VM: Ventilatory Mask; T: Temperature; HR: Heart Rate; OR: Operative Room

\section{Introduction}

Coronavirus disease (CoVid-19) started in December 2019 in Wuhan, Hubei province, China [1,2] and the World Health Organization declared a global health emergency on January 30, 2020 [3]. Interhuman transmission has resulted in 73,451 cases in 26 countries with 1,875 deaths as of February 2020 [4]. Some early investigators have observed radiologic patterns on Chest X - Ray (C-XR) and Computed Tomography (CT) scan of patients affected from CoVid-19 pneumonia $[5,6]$.

An initial prospective analysis in Wuhan revealed bilateral lung opacities on 40 of $41(98 \%)$ chest CT in infected patients and described lobular and subsegmental areas of consolidation as the most typical findings [7]. Other investigators examined chest CT scan in 21 CoVid-19 patients with pneumonia and found high rates of ground glass opacities and consolidation, sometimes with a rounded morphology and peripheral lung distribution [8]. Another group evaluated lung abnormalities on CT scan related to disease course and found the most extensive lung damage approximately ten days after symptom onset [9]. Radiology represents a fundamental evaluation in these patients to ensure timely treatment. Eventually, as infection turns from acute and subacute phases to either a completely healed outcome or to a chronic condition, radiologic imaging should be investigated to evaluate if long-term complications can occur.

\section{Case Report}

A 69 years old lady was admitted for medical treatment on $27^{\text {th }}$ March 2020 because of fever and dry cough lasting 10 days and not responsive to Azithromycin for 3 days. She suffered from obesity, hypertension, previous melanoma, hysterectomy, colonic diverticula, gastric leiomyoma and left lower lobe lobectomy for lung cancer. Clinical evaluation at admittance was: tachypnea, weakened murmur on the right 


\section{Open Access Journal of Pulmonary \& Respiratory Sciences}

chest and abdominal tenderness. C-XR showed large pleural effusion on the right side with thickness of the right lower lobe, and bilateral interstitial reinforcement. Parameters were: Sat02 94\% with $0212 \mathrm{~L} / \mathrm{min}$ and Ventilatory Mask (VM) $60 \%$, Temperature (T) $37^{\circ} \mathrm{C}$ and Heart Rate (HR) 94 pulses. Patient was found positive to CoVid-19 and referred to Pulmonology. On the 1st day of hospitalization arterial blood gas parameters were: $\mathrm{PaO} 276 \mathrm{mmHg}$; PaCo2 35 mmHg; pH 7,53; Sat02 95\% with $0212 \mathrm{~L} / \mathrm{min}$ and VM 60 $\%$; T $37,6^{\circ} \mathrm{C}$. Therapy was as follows: Danuravir, Ritonavir, Hydroxyclorochin, Enoxaparin, Lasix and Kanrenol. On the $2^{\text {nd }}$ day of recovery she presented Sat02 $97 \%$ with VM 60 $\%$ and $0212 \mathrm{~L} / \mathrm{min}$, TC $37,4^{\circ} \mathrm{C}$. On the $3^{\text {rd }}$ day Sat $0297 \%$ with VM $60 \%$ and $0212 \mathrm{~L} / \mathrm{min}, \mathrm{T} 37,2^{\circ} \mathrm{C}$. On the $4^{\text {th }}$ day Sat $0297 \%$ with VM $50 \%$ and $0212 \mathrm{~L} / \mathrm{min}$, no fever. CT scan showed a huge pleural effusion on the right side with an almost complete lung collapse, in which ground-glass areas were evaluable. Multiple ground-glass areas were also evaluable on the left lung (Figure 1).



Because of the suspect of pleural empyema, she was referred to our Department and the day after she went to Operative Room (OR). Blood counts at admittance were: WBC 14.69/mm3, Hb $123 \mathrm{~g} / \mathrm{L}$, INR 1.13, PTT $28.4 \mathrm{sec}$, Na $134 \mathrm{mEq} / \mathrm{L}, \mathrm{K} 4.2 \mathrm{mEq} / \mathrm{L}$, Ca 7,6 mEq/L, nitrogen $10 \mathrm{mg} /$ $\mathrm{dL}$, creatinine $0,46 \mathrm{mg} / \mathrm{dL}$, PCR $9.11 \mathrm{mg} / \mathrm{dL}$. Double lumen tube placement was difficult for obesity and short neck. Anesthesiological maneuvers were performed quickly. VATS has been performed using 4 accesses and $30^{\circ}$ camera. After lung collapsed a great amount of fluid has been removed and was sent for microbiology examination. Despite of CT scan pattern, clinical condition and laboratory findings, color, smell and consistence of the fluid no bacteria have been found within it. Pleural space appeared full of adhesions from apex to diaphragm and the main effort for surgeons was to free the lung. Separation of lobes resulted difficult because of thickness of the fissure. Pleural biopsies have been taken on the parietal pleura and sent as a specimen for Pathologist, whose diagnosis was fibrinoid acute pleuritis with abscess. Pleural space has been repeatedly washed until saline solution went into clear. Two $28 \mathrm{Fr}$ chest tubes have been placed in the apex and another one right above the diaphragm. More than satisfactory was the lung re-expansion at the end of intervention (Figure 2).

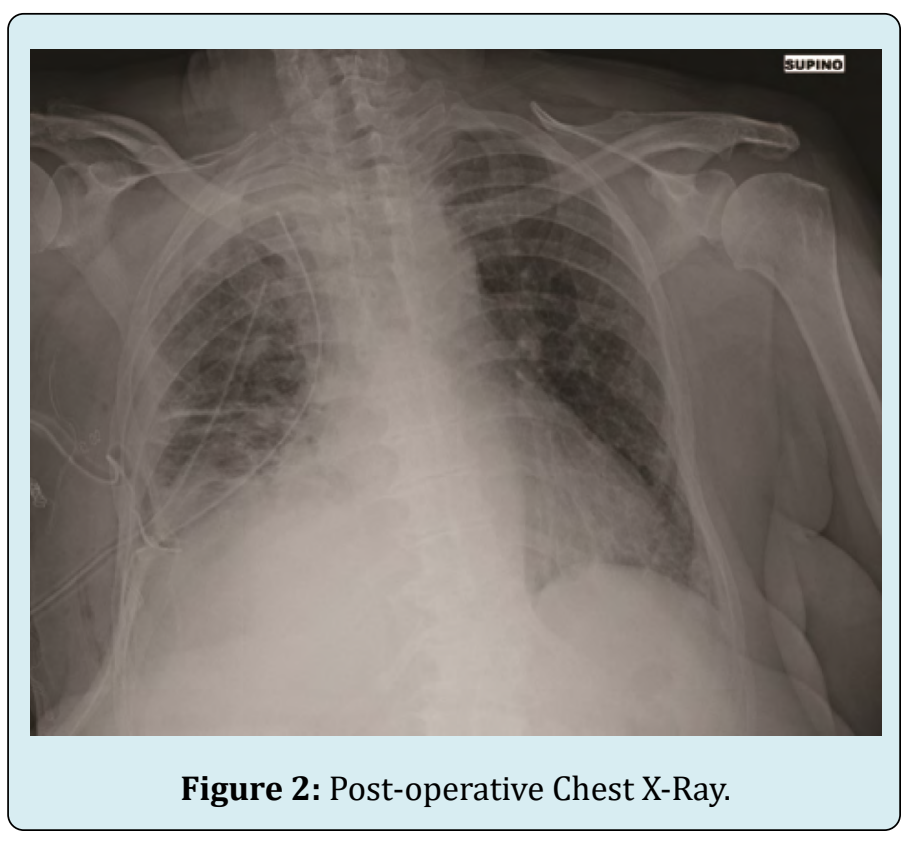

After surgery the patient was admitted to Intensive Care Unit. Blood counts after intervention were: WBC 13.29/mm3, $\mathrm{Hb} 91 \mathrm{~g} / \mathrm{dL}$, Na $134 \mathrm{mEq} / \mathrm{L}, \mathrm{K} 4.1 \mathrm{mEq} / \mathrm{L}$, Ca $7.9 \mathrm{mg} / \mathrm{dL}$, nitrogen $10 \mathrm{mg} / \mathrm{dL}$, creatinine $0.37 \mathrm{mg} / \mathrm{dL}, \mathrm{PCR} 11,10$. After 3 days in Intensive Care Unit patient has been discharged and re-sent to Pneumology Unit. On $15^{\text {th }}$ post-operative day patient was doing better and was discharged after resulting CoVid-19 negative.

\section{Comment}

The interest of this case is that a CoVid-19 pneumonia complicated with a huge pleural effusion and the patient had benefit from surgery. Moreover, this patient suffered from 


\section{Open Access Journal of Pulmonary \& Respiratory Sciences}

two different neoplasms (melanoma and lung cancer) so that it is reasonable to retain that a somewhat immunologic disorder represented a fundamental comorbidity for the onset of CoVid-19 pneumonia. Nonetheless it is noteworthy how CT findings correlate with clinical and laboratory findings. Wu et. al. found that ground-glass opacity was the most common CT manifestation in the fourth stage of CoVid-19 [10], while pleural effusion was found in severe patients, which mainly occurred in stage 2 of the disease course, even if only with a small amount in most cases. In the study of $\mathrm{Wu}$ et al. pleural effusion may be somewhat associated with chronic underlying diseases. Pleural effusion frequency decreases in stage 3 and stage 4 , that is when the initial alveolar inflammatory damage turns into fibrousmucous like exudate at the end stage of CoVid-19 pneumonia. The present case is reasonably to be referred to a stage $2 / 3$ disease. Probably a thoracentesis or a chest tube should have represented the best proceeding, if done at the beginning of pleural effusion onset. In all cases of pleural effusion infact it is mandatory to maintain a close follow - up with C - XR and/ or CT scan to avoid further complications like in this patient. If pleural effusion doesn't disappear despite of conservative therapy, it is recommended to perform a thoracentesis and/ or to put a chest drain before sepsis occurs. But if patient presents with pleural effusion and sepsis, and expecially if patient as a CoVid-19 pneumonia, in our opinion surgical clearance of pleural space becomes mandatory all means also without previous thoracentesis or chest drain.

Disclosure Statement: The authors have nothing to disclose.

\section{References}

1. Zhu N, Zhang D, Wang W, Li X, Yang B, et al. (2019) A novel coronavirus from patients with pneumonia in China. N. Engl J Med 2020 382: 727-733.
2. Tan WJ, Zao X, Ma XJ, Wang W, Niu P, et al. (2020) A novel coronavirus genome identified in a cluster of pneumonia case - Wuhan, China 2019 - 2020. China CDC Weekly 2(4): 61-62.

3. Mahase E (2020) China coronavirus: WHO declares international emergency as death toll exceeds 200. BMJ 368: $\mathrm{m} 408$.

4. Chan JF, Yuan S, Kok KH, Kelvin KW, Chu H, et al. (2020) A familiar cluster of pneumonia associated with the 2019 novel coronavirus indicating person to person transmission: a study of a family cluster. Lancet 395(10223): 514-523.

5. Phelan AL, Katz R, Gostin LO (2020) The Novel Coronavirus Originating in Wuhan, China: Challenges for Global Health Governance. JAMA 323(8):709-710.

6. Li X, Zeng X, Liu B, Yu Y (2020) COVID - 19 Infection presenting with CT halo sign. Radiology: Cardiothoracic Imaging 2(1): 1.

7. Huang C, Wang Y, Li X, Ren L, Zhao J, et al. (2020) Clinical features of patients infected with 2019 novel coronavirus in Wuhan, China. Lancet 395(10223): 497-506.

8. Chung M, Bernheim A, Mei X, Zhang N, Huang M, et al. (2020) CT Imaging Features of 2019 Novel Coronavirus (2019-nCoV). Radiology 295(1): 202-207.

9. Pan F, Ye T, Sun P, Gui S, Liang B, et al. (2020) Time Course of Lung Changes On Chest CT During Recovery from 2019 Novel Coronavirus (COVID-19) Pneumonia. Radiology.

10. Lan W, Ran Y, Dajing G, (2020) Longitudinal changes of chest appearances in patients with severe COVID - 19 and its correlation with laboratory examination results. European Journal of Radiology Open. 